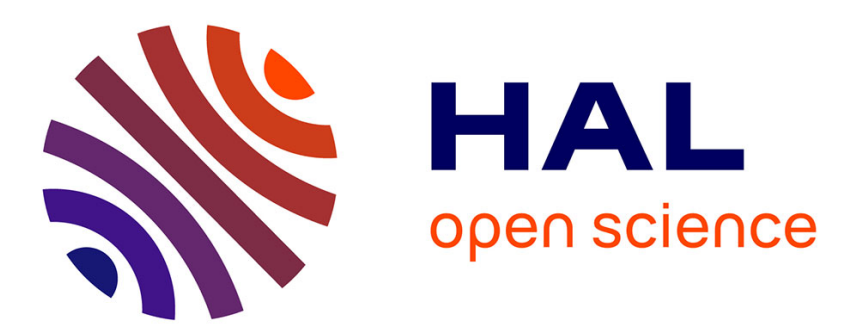

\title{
Résonances d'ondes d'interface de coques cylindriques minces immergées : détermination et interprétation
}

\author{
A. Gérard, J.L. Rousselot, J.L. Izbicki, G. Maze, J. Ripoche
}

\section{To cite this version:}

A. Gérard, J.L. Rousselot, J.L. Izbicki, G. Maze, J. Ripoche. Résonances d'ondes d'interface de coques cylindriques minces immergées: détermination et interprétation. Revue de Physique Appliquée, 1988, 23 (3), pp.289-299. 10.1051/rphysap:01988002303028900 . jpa-00245773

\section{HAL Id: jpa-00245773 https://hal.science/jpa-00245773}

Submitted on 1 Jan 1988

HAL is a multi-disciplinary open access archive for the deposit and dissemination of scientific research documents, whether they are published or not. The documents may come from teaching and research institutions in France or abroad, or from public or private research centers.
L'archive ouverte pluridisciplinaire HAL, est destinée au dépôt et à la diffusion de documents scientifiques de niveau recherche, publiés ou non, émanant des établissements d'enseignement et de recherche français ou étrangers, des laboratoires publics ou privés. 
Classification

Physics Abstracts

$43.20 \mathrm{~F}-43.20 \mathrm{~K}-43.30 \mathrm{~J}$

\title{
Résonances d'ondes d'interface de coques cylindriques minces immergées : détermination et interprétation
}

\author{
A. Gérard $\left({ }^{1}\right)$, J. L. Rousselot $\left({ }^{2}\right)$, J. L. Izbicki $\left({ }^{3}\right)$, G. Maze $\left({ }^{3}\right)$ et J. Ripoche $\left({ }^{3}\right)$ \\ ( $\left.{ }^{1}\right)$ Université de Bordeaux I, Laboratoire de Mécanique Physique, UA 867, 351 Cours de la Libération, \\ 33405 Talence Cedex, France \\ ( $\left.{ }^{2}\right)$ Le Centre Thomson d'Applications Radar, 18-20 Rue Grange Dame Rose, BP 40, 78141 Vélizy \\ Villacoublay Cedex, France \\ $\left({ }^{3}\right)$ Université du Havre, Laboratoire d'Electronique et d'Automatique (Ultrasons), Place R. Schuman, 76610 \\ Le Havre, France
}

(Reçu le 20 juillet 1987, révisé le 11 décembre 1987, accepté le 16 décembre 1987)

\begin{abstract}
Résumé. - L'étude expérimentale et théorique de coques cylindriques minces, remplies d'air et immergées dans l'eau, met en évidence l'existence de résonances liées à des ondes dont la vitesse de phase est inférieure à celle d'une onde acoustique dans l'eau. Des résultats concernant des tubes dont le rapport entre le rayon interne et le rayon externe varie entre 0,93 et 0,99 sont présentés. L'accord entre les fréquences des résonances mesurées et calculées est très bon. Ces résonances n'existent que dans une bande limitée de fréquence pour un tube donné ; ceci est lié à la largeur des résonances. Une interprétation théorique est donnée de la fréquence de coupure haute.
\end{abstract}

\begin{abstract}
The experimental and theoretical studies of thin cylindrical air-filled shells immersed in water show the existence of resonances connected with waves which the phase velocity is inferior to the phase velocity of the sound in the water. Results which are presented concern shells with the ratio between the inner and the outer radius varying between 0.93 and 0.99 . The agreement between the measured frequency resonance and the calculated one is quite good. These resonances appear only in a frequency range for a given shell ; this fact is connected with the resonance width. The high frequency cut is theoretically explained.
\end{abstract}

\section{Introduction.}

La diffusion d'une onde plane monochromatique ultrasonore par un tube immergé dans l'eau a fait l'objet de nombreux travaux au cours de ces dernières années. Les résultats obtenus ne peuvent s'expliquer que par l'existence d'ondes se propageant à la périphérie du diffuseur. Sur le plan théorique, deux approches sont utilisées pour décrire et interpréter les ondes circonférentielles se propageant à la périphérie des objets insonés : la théorie de la matrice $S$ et la transformation de Sommerfeld-Watson [1]. Ces ondes constituent, lorsque la fréquence de l'onde incidente est égale à l'une des fréquences propres de la coque plongée dans l'eau, un système d'ondes stationnaires. On dit qu'une résonance du diffuseur est excitée; le mode de vibration $n$ peut être déterminé expérimentalement par la Méthode d'Isolement et d'Identification des Résonances [2]. La concordance des approches théoriques, d'une part, et des résultats numériques et expérimentaux, d'autre part, a été vérifiée pour les cylindres pleins et les tubes dont la paroi est relativement épaisse [34].

Des résultats numériques récents [5] concernant un tube tel que $b / a=0,85$ ( $b$ désigne le rayon intérieur et $a$ le rayon extérieur) rempli d'air et plongé dans l'eau ont mis en évidence la présence d'une onde qui n'était pas apparue antérieurement : elle a été dénommée $l=0$. Une des caractéristiques de cette onde est que sa vitesse de phase est inférieure à celle d'une onde acoustique dans l'eau. Une telle onde a été mise en évidence expérimentalement [6].

Cet article a pour objet, d'une part, d'apporter de nouveaux résultats théoriques et expérimentaux concernant des tubes tels que $0,93 \leqslant b / a \leqslant 0,99$ et, d'autre part, de justifier l'existence d'une onde circonférentielle dans une bande de fréquence limitée. 


\section{Résultats expérimentaux.}

Les tubes d'aluminium utilisés ont un diamètre intérieur de $20 \mathrm{~mm}$. Le rapport $\eta=b / a$ entre le rayon interne $b$ et le rayon externe $a$ évolue entre 0,93 et 0,99 à 0,001 près. Les tubes, de longueur $20 \mathrm{~cm}$, remplis d'air sont immergés verticalement dans l'eau. Pour les tubes tels que $\eta$ est égal à 0,93 , $0,95,0,97,0,98$, une méthode quasi-harmonique est utilisée. Des trains d'onde acoustique de durée $300 \mu \mathrm{s}$ et de fréquence de récurrence $3 \mathrm{kHz}$ sont émis par des transducteurs ultrasonores large bande placés à $1 \mathrm{~m}$ environ du tube. Le tube n'est insoné que sur une partie de sa hauteur. Suivant les expériences, des transducteurs de fréquence centrale $1 \mathrm{MHz}$ ou 2,25 $\mathrm{MHz}$ sont utilisés.

La Méthode d'Isolement et d'Identification des Résonances est mise en jeu [2]. L'amplitude du signal rétrodiffusé par le tube peut être analysée, en fonction de la fréquence, soit dans le régime forcé on obtient alors le spectre de rétrodiffusion - , soit dans le régime libre - on obtient alors le spectre du régime libre ou spectre des résonances - Les spectres sont tracés en fonction de la fréquence

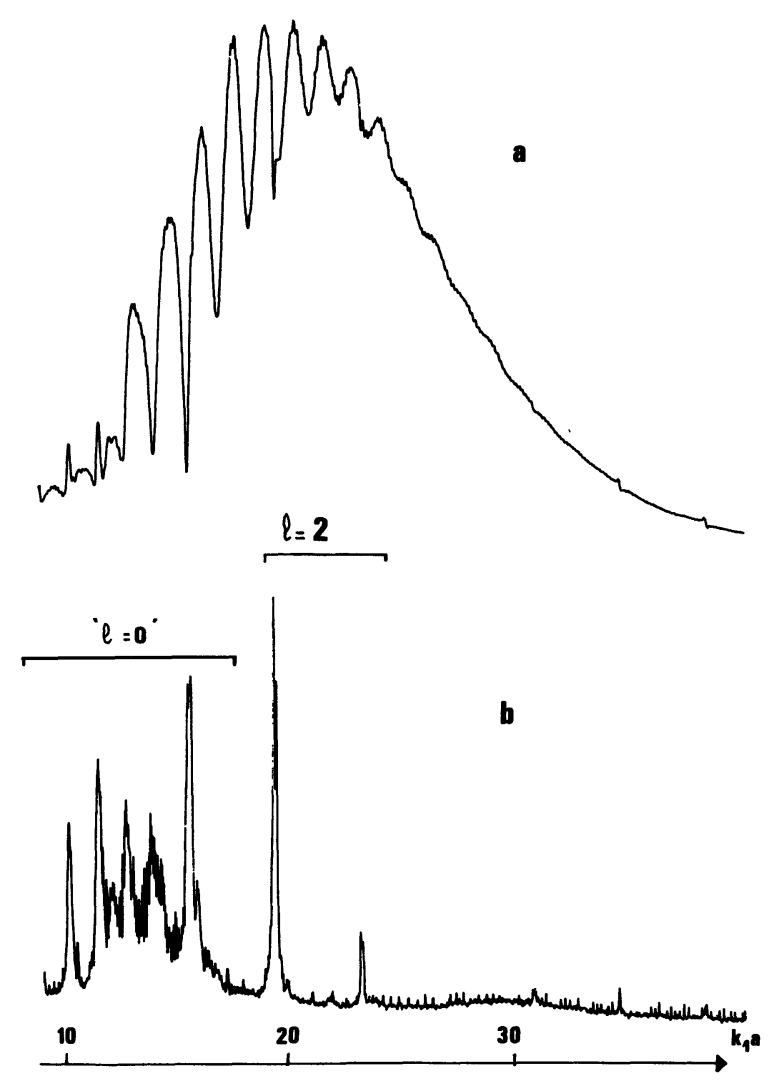

Fig. 1. - Tube $b / a=0,93$, a) Spectre du régime forcé, b) Spectre des résonances.

[Cylindrical Shell $b / a=0.93$, a) Steady state spectrum, b) Resonance spectrum.] réduite $x_{1}=k_{1} a\left(k_{1}\right.$ désigne la norme du vecteur d'onde de l'onde incidente). La bande passante des transducteurs n'a pas été corrigée. Pour les tubes précédents, le spectre de rétrodiffusion présente, pour une certaine gamme de fréquence, des « oscillations » de grande amplitude et serrées (Figs. 1-4). Par exemple, pour le tube correspondant à $\eta=0,93$, on observe ces oscillations pour les $x_{1}$ compris entre 10 et 30 environ. Celles-ci correspondent, dans le spectre des résonances (Figs. 1-4) à des pics relativement larges, en tout cas plus larges que les pics correspondant aux résonances d'ondes de galerie à écho du type $l=2$ bien connues [4]. Ces résonances peuvent être identifiées [2]. Aux fréquences de résonances, il y a génération d'un système d'ondes stationnaires autour du tube. Pour ces fréquences, un transducteur positionné sur un bras tournant dans un plan horizontal contenant l'axe du faisceau incident, va permettre l'enregistrement d'un diagramme angulaire de rayonnement correspondant à un mode de vibration particulier. Il s'agit donc d'étudier la réémission libre dans l'eau de l'onde stationnaire. La succession des maxima et des minima (Figs. 5-8) correspond respectivement aux

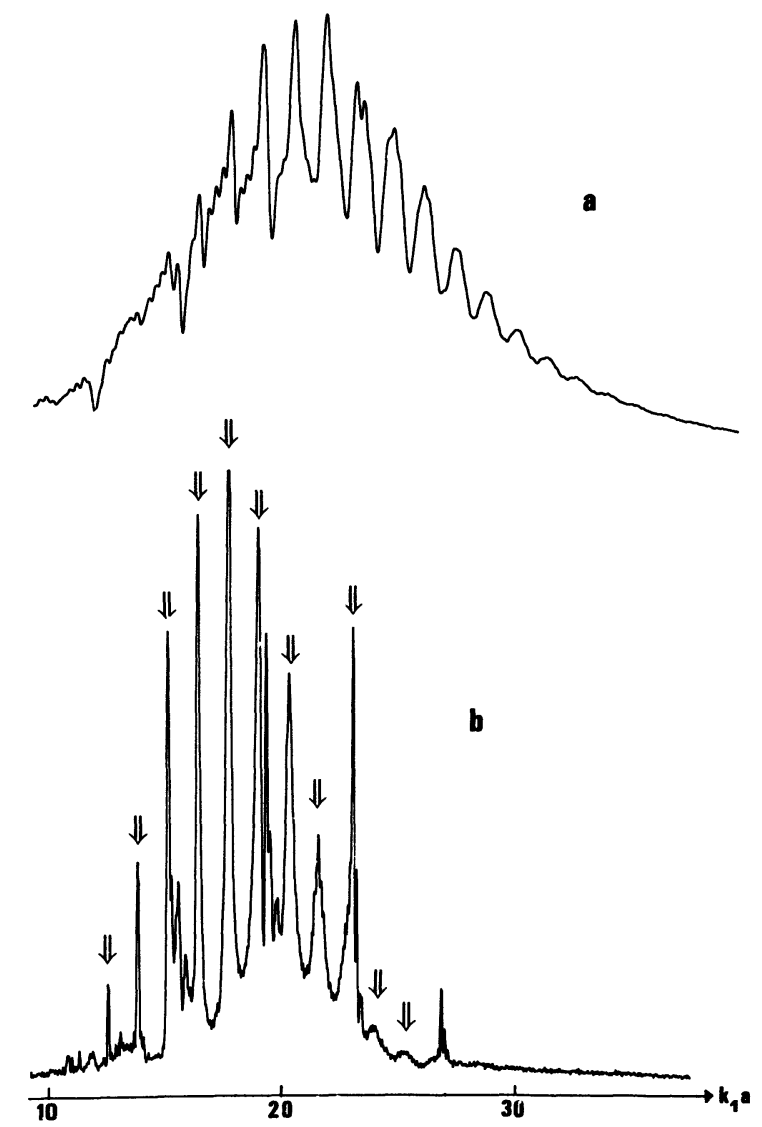

Fig. 2. - Tube $b / a=0,95$, a) Spectre du régime forcé, b) Spectre des résonances : $« l=0 »$.

[Cylindrical shell $b / a=0.95$, a) Steady state spectrum, b) Resonance spectrum.] 


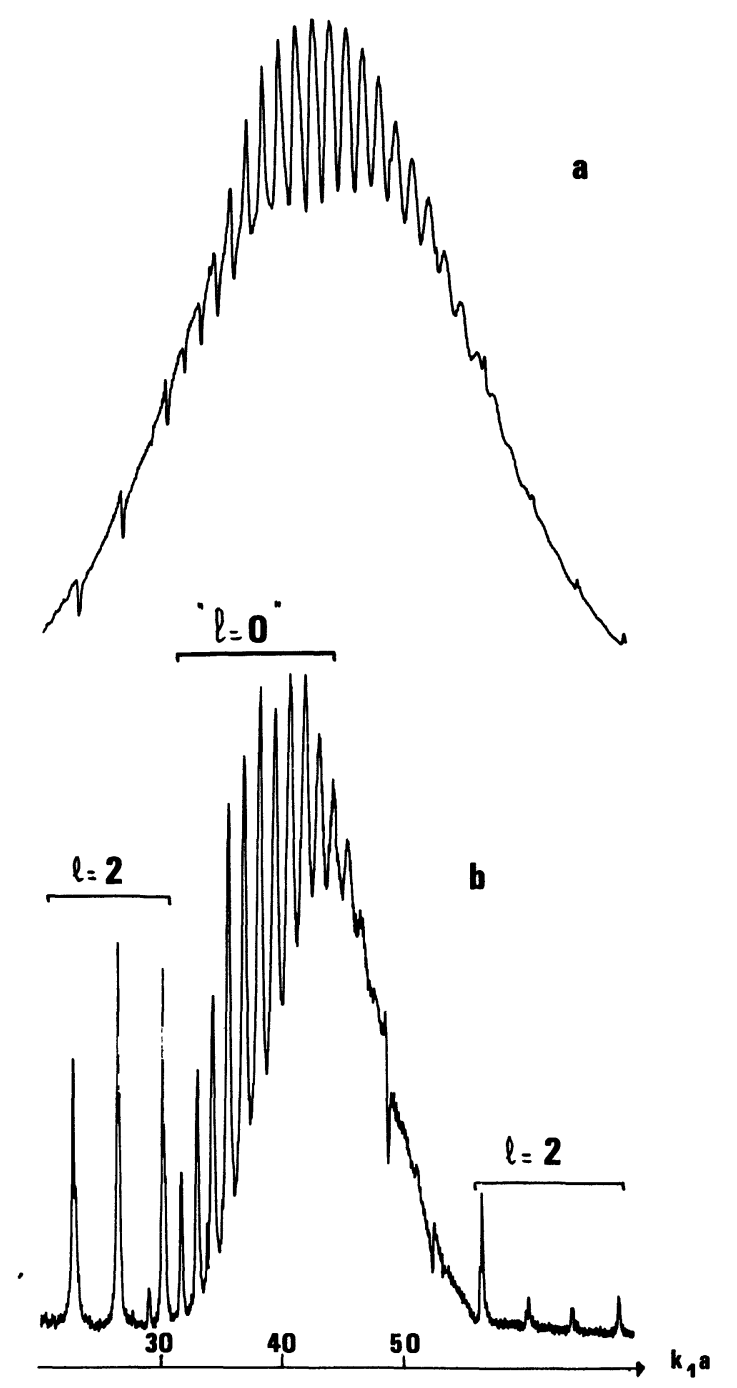

Fig. 3. - Tube $b / a=0,97$, a) Spectre du régime forcé, b) Spectre des résonances.

[Cylindrical shell $b / a=0.97$, a) Steady state spectrum, b) Resonance spectrum.]

ventres et aux nœuds de vibration. La valeur $n \mathrm{du}$ mode de vibration est égal à la moitié du nombre de maxima.

On constate que plus le tube est mince et plus les résonances observées sont de modes élevés et donc le relevé des maxima de réémission est plus délicat. L'ensemble des résultats obtenus expérimentalement est reporté dans les tableaux I-IV. Ces modes ne correspondent pas à des modes propres du tube libre [7]. Ces résonances n'apparaissent que pour une certaine gamme de fréquence et pour certains modes. Lorsque le rapport $\boldsymbol{\eta}$ tend vers l'unité, l'ensemble des modes observés est décalé vers les hautes fréquences et les modes d'ordre élevé. Pour toutes ces résonances, la vitesse de phase des ondes correspondantes, obtenue à partir de la relation:

$$
c_{l=0}=c_{1} \frac{k_{1} a}{n}
$$

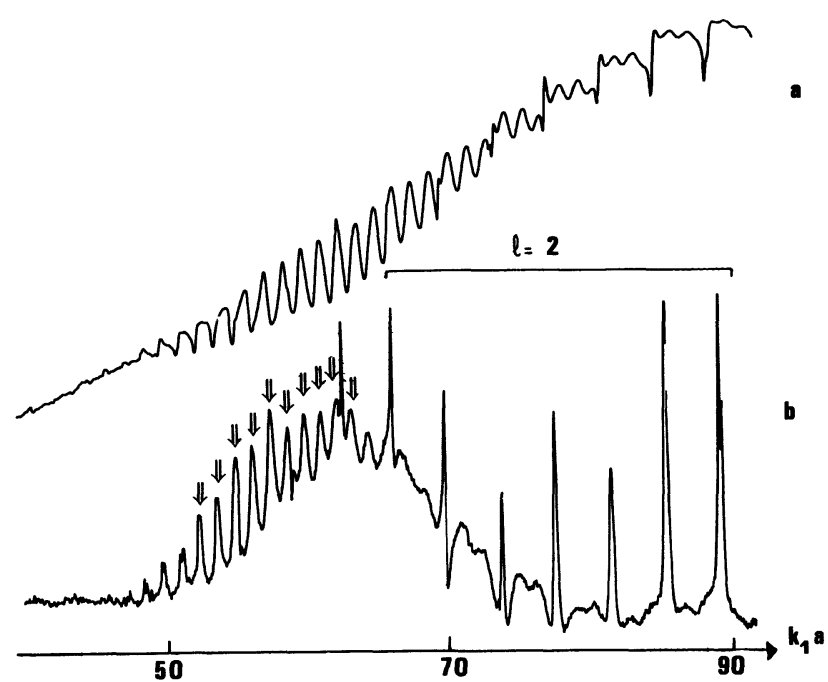

Fig. 4. - Tube $b / a=0,98$, a) Spectre du régime forcé, b) Spectre des résonances : $« l=0 »$.

[Cylindrical shell $b / a=0.98$, a) Steady state spectrum, b) Resonance spectrum.]

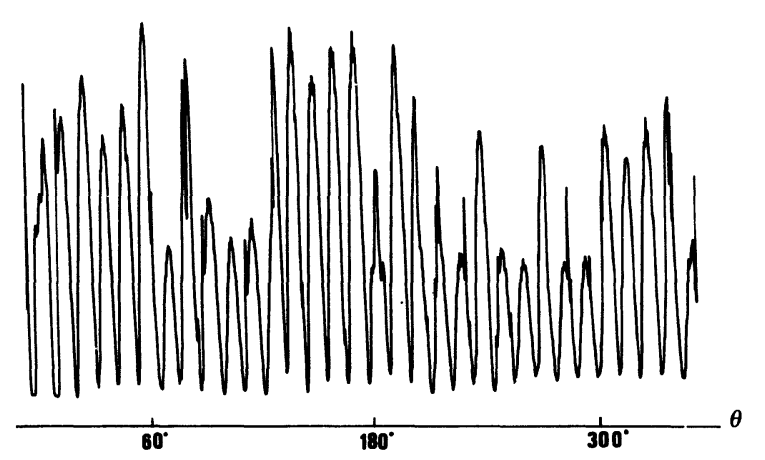

Fig. 5. - Tube $b / a=0,93$. Identification de la résonance $n=16, k_{1} a=13,20$.

[Cylindrical shell $b / a=0.93$. Resonance identification $n=16, k_{1} a=13.20$.]

(où $c_{1}$ désigne la vitesse de phase d'une onde acoustique dans l'eau), conduit à des vitesses de phase toujours inférieure à $c_{1}$. Des constatations semblables ont été obtenues sur des tubes d'aluminium remplis d'air dont le rapport b/a est compris entre 0,67 et 0,90 [6]. L'évolution de la gamme de fréquences et des modes observés est beaucoup plus importante entre 0,90 et 0,98 qu'entre 0,67 et 0,90 . Ceci est bien visualisé sur la courbe de Regge (Fig. 9).

Il est également remarquable que la présence de ces résonances se fait sentir dans une gamme plus importante de fréquence dans le spectre du régime forcé que dans le spectre des résonances.

Pour observer un phénomène analogue avec un tube de rapport $b / a=0,99$, il est nécessaire d'augmenter la fréquence de l'onde incidente. L'atté- 


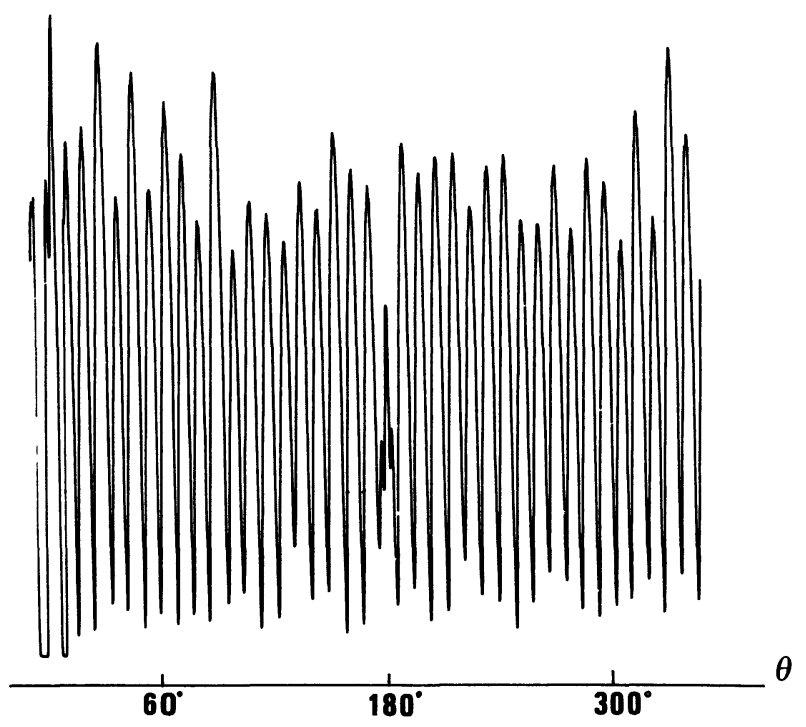

Fig. 6. - Tube $b / a=0,95$. Identification de la résonance $n=20, k_{1} a=15,15$.

[Cylindrical shell $b / a=0.95$. Resonance identification $n=20, k_{1} a=15.15$.]
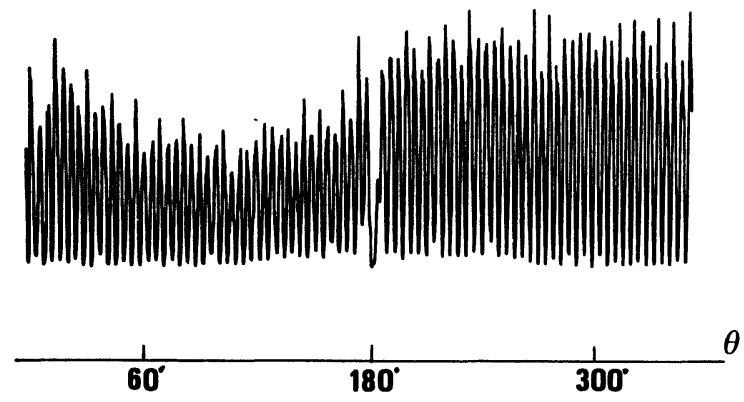

Fig. 7. - Tube $b / a=0,97$. Identification de la résonance $n=42, k_{1} a=36,70$.

[Cylindrical shell $b / a=0.97$. Resonance identification $n=42, k_{1} a=36.70$.]

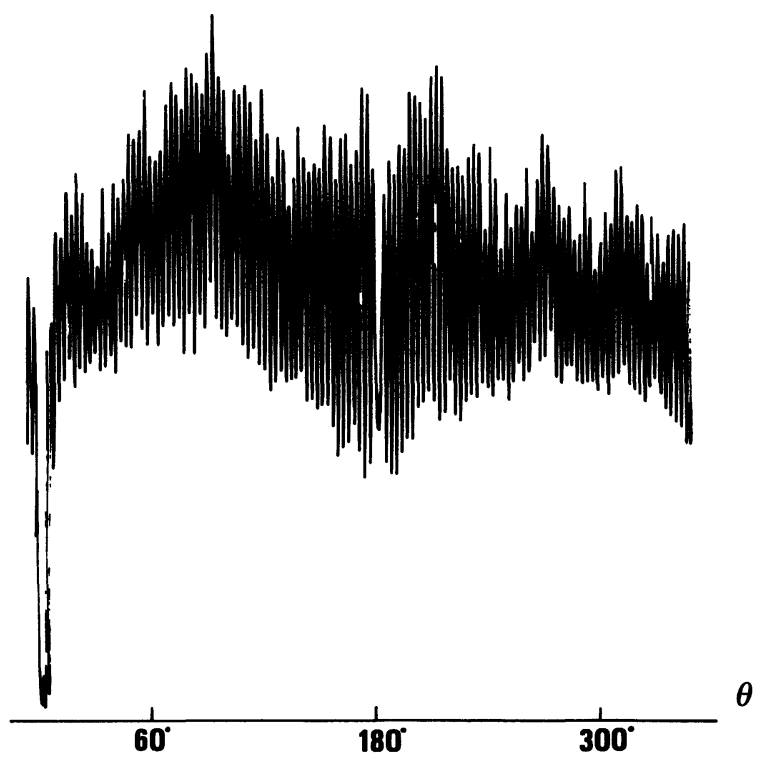

Fig. 8. - Tube $b / a=0,98$. Identification de la résonance $n=63, k_{1} a=56,76$.

[Cylindrical shell $b / a=0.98$. Resonance identification $n=63, k_{1} a=56.76$.]

nuation des ondes ultrasonores se propageant dans l'eau croît comme le carré de la fréquence ; il est nécessaire de rapprocher le transducteur de la cible. Il faut aussi diminuer la durée du train de sinusoïdes incident, ce qui conduit à diminuer l'énergie acoustique reçue par le tube. Les mesures sont alors délicates. Une méthode quasi-impulsionnelle permet la production d'ondes ultrasonores avec une énergie plus grande qu'avec une méthode quasi-harmonique. Ainsi, pour le tube $\eta=0,99$, le transducteur est excité par une impulsion de durée $0,3 \mu$ s d'amplitude $300 \mathrm{~V}$. A la réception, le signal est composé par un premier écho correspondant à la réflexion spéculaire et une suite d'échos correspondant au caractère élastique de la cible [8-11]. L'analyse de la totalité

Tableau I. - TUBE $b / a=0,93$.

[TUBE $b / a=0.93$.]

$\begin{array}{cccccc}n & k_{1} a \text { théorique } & k_{1} a \text { expérimental } & \frac{c_{l=0}}{c_{1}} \text { théorique } & \frac{c_{l=0}}{c_{1}} \text { expérimental } & \Gamma_{n l} \\ 12 & 8,06 & 8,04 & 0,67 & & 0,67 \\ 13 & 9,32 & 9,27 & 0,72 & 0,71 & 0,014 \\ 14 & 10,61 & 10,59 & 0,76 & 0,76 & 0,080 \\ 15 & 11,91 & 11,96 & 0,79 & 0,80 & 0,162 \\ 16 & 13,20 & 13,20 & 0,82 & 0,82 & 0,277 \\ 17 & 14,48 & 14,40 & 0,85 & 0,85 & 0,462 \\ 18 & 15,74 & 15,80 & 0,87 & 0,88 & 0,694 \\ 19 & 16,96 & 17,30 & 0,89 & 0,91 & 0,964\end{array}$


Tableau II. $-T U B E b / a=0,95$.

[TUBE $b / a=0.95$.

\begin{tabular}{cccccc}
\hline$n$ & $k_{1} a$ théorique & $k_{1} a$ expérimental & $\frac{c_{l=0}}{c_{1}}$ théorique & $\frac{c_{l=0}}{c_{1}}$ expérimental & $\Gamma_{n l}$ \\
18 & 12,72 & 12,53 & 0,71 & 0,70 & 0,014 \\
19 & 13,92 & 13,83 & 0,73 & 0,73 & 0,036 \\
20 & 15,31 & 15,15 & 0,77 & 0,76 & 0,032 \\
21 & 16,62 & 16,46 & 0,79 & 0,78 & 0,053 \\
22 & 17,93 & 17,80 & 0,81 & 0,81 & 0,103 \\
23 & 19,24 & 19,0 & 0,84 & 0,83 & 0,195 \\
24 & 20,54 & 20,32 & 0,86 & 0,85 & 0,348 \\
25 & 21,84 & 21,10 & 0,87 & 0,84 & 0,578 \\
26 & 23,13 & 22,58 & 0,89 & 0,87 & 0,737 \\
27 & 24,45 & 23,92 & 0,90 & 0,89 & 1,371 \\
28 & 25,79 & 25,17 & 0,92 & 0,90 &
\end{tabular}

Tableau III. - TUBE $b / a=0,97$.

[TUBE $b / a=0.97$.

$\begin{array}{cccccc}n & k_{1} a \text { théorique } & k_{1} a \text { expérimental } & \frac{c_{l=0}}{c_{1}} \text { théorique } & \frac{c_{l=0}}{c_{1}} \text { expérimental } & \Gamma_{n l} \\ 39 & 32,90 & 32,90 & 0,84 & 0,84 & 0,054 \\ 40 & 34,21 & 34,10 & 0,85 & 0,85 & 0,086 \\ 41 & 35,51 & 35,50 & 0,87 & 0,87 & 0,126 \\ 42 & 36,80 & 36,70 & 0,87 & 0,87 & 0,192 \\ 43 & 38,07 & 38,12 & 0,88 & 0,89 & 0,272 \\ 44 & 39,34 & 39,30 & 0,89 & 0,89 & 0,379 \\ 45 & 40,59 & 40,76 & 0,90 & 0,90 & 0,539 \\ 46 & 41,84 & 41,70 & 0,91 & 0,91 & 0,760 \\ 47 & 43,08 & 43,50 & 0,92 & 0,92 & 1,022 \\ 48 & 44,31 & 44,38 & 0,93 & 0,92 & 1,226\end{array}$

Tableau IV. - TUBE $b / a=0,98$.

[TUBE $b / a=0.98$.]

$\begin{array}{lccccc}n & k_{1} a \text { théorique } & k_{1} a \text { expérimental } & \frac{c_{l=0}}{c_{1}} \text { théorique } & \frac{c_{l=0}}{c_{1}} \text { expérimental } & \Gamma_{n l} \\ 60 & 51,30 & 53,01 & 0,85 & 0,88 & 0,012 \\ 61 & 52,62 & 54,36 & 0,86 & 0,89 & 0,017 \\ 62 & 53,95 & 55,71 & 0,87 & 0,90 & 0,033 \\ 63 & 55,25 & 56,76 & 0,88 & 0,90 & 0,049 \\ 64 & 56,54 & 58,37 & 0,88 & 0,91 & 0,080 \\ 65 & 57,84 & 59,50 & 0,89 & 0,91 & 0,097 \\ 66 & 59,11 & 61,33 & 0,90 & 0,93 & 0,131 \\ 67 & 60,37 & 62,77 & 0,90 & 0,94 & 0,164 \\ 68 & 61,63 & 64,30 & 0,90 & 0,95 & 0,254 \\ 69 & 62,87 & 65,30 & 0,91 & 0,95 & 0,298\end{array}$

du signal reçu par un analyseur de spectres fournit un spectre analogue au spectre de rétrodiffusion forcée (Fig. 10). On remarque des oscillations analo- gues à celles précédemment obtenues pour la bande de fréquence réduite 125-170. Une fois connue la bande de fréquence "intéressante », la méthode 


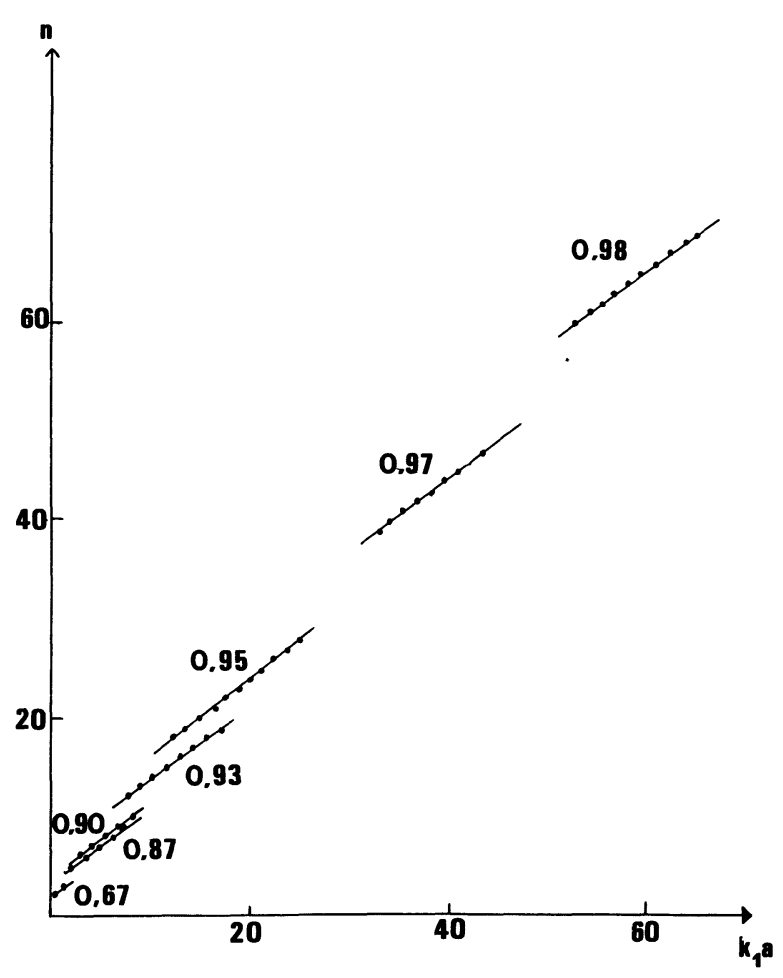

Fig. 9. - Trajectoire de Regge.

[Regge trajectory.]

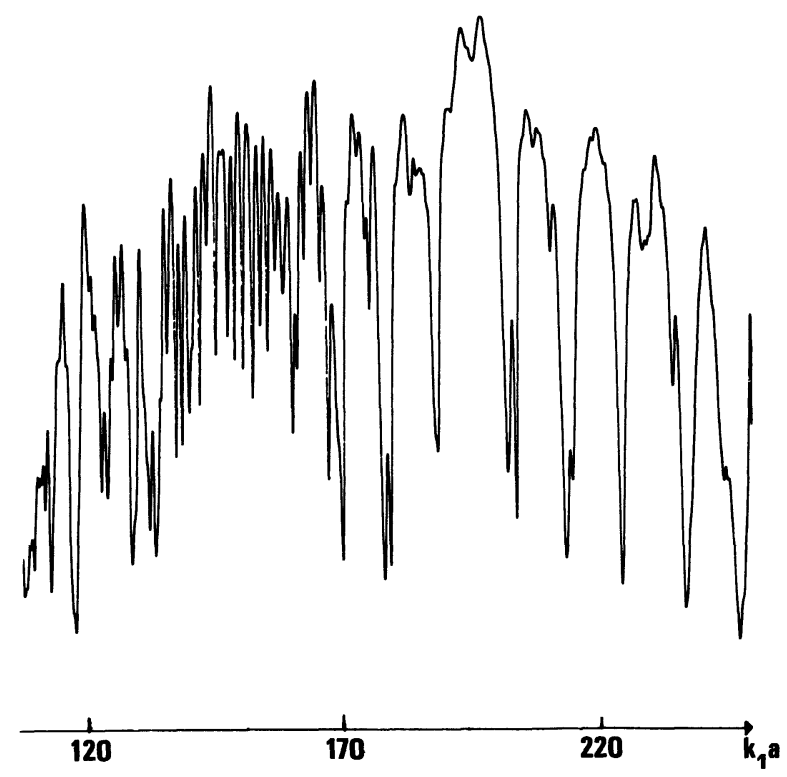

Fig. 10. - Tube $b / a=0,99$. Spectre du signal rétrodiffusé.

[Cylindrical shell $b / a=0.99$. Backscattered signal spectrum.]

quasi-harmonique a été essayée. Deux résonances ont été localisées pour $k_{1} a=134,0$ et pour $k_{1} a=141,1$. L'identification des résonances ne donne pas un résultat suffisamment précis. En effet, pour des raisons de positionnement mécanique des transducteurs, il est exclu d'effectuer un tour complet de la cible. Une rotation partielle est possible mais le relevé des maxima n'est pas aisé (il y en a un pour une rotation de 1,2 degré environ).

Les résultats précédents sont obtenus lorsque le tube est immergé dans l'eau pendant un temps suffisant. Cette constatation, jointe au fait que les résonances observées ne correspondent pas à des modes propres de la coque libre, incite à penser que l'onde $« l=0$ » est sensible au liquide externe : c'est une onde d'interface.

\section{Etude de la fréquence limite d'observation.}

Il s'agit de proposer une première interprétation à l'effet de fenêtre et en particulier à la fréquence maximale de l'observation de l'onde $« l=0$ ». L'ensemble des résultats expérimentaux disponibles à ce jour concerne les tubes de rapport $\eta=0,67$; 0,$75 ; 0,85 ; 0,88 ; 0,90$ qui ont déjà été étudiés [6] et des tubes de rapport $\eta=0,93 ; 0,95 ; 0,97 ; 0,98$ qui font l'objet du présent article. L'interprétation est obtenue à partir de considérations asymptotiques sans effectuer le calcul explicite des fréquences des résonances. Cependant, le calcul des fréquences des résonances et les résultats sont rapportés au paragraphe suivant.

Dans un milieu 1 liquide, on considère une onde plane incidente rencontrant une coque élastique cylindrique de rayon extérieur $a$ et de rayon intérieur $b$. On pose $\eta=b / a$. Cette coque est remplie d'air. On se propose de déterminer le champ de pression rayonné dans le milieu 1. En théorie des petites perturbations, les équations régissant le mouvement de chaque milieu sont du type :

$$
\frac{\partial^{2} F}{\partial r^{2}}+\frac{1}{r} \frac{\partial F}{\partial r}+\frac{1}{r^{2}} \frac{\partial^{2} F}{\partial \theta^{2}}=\frac{1}{c^{\prime 2}} \frac{\partial^{2} F}{\partial t^{2}}
$$

où $c^{\prime}$ désigne la vitesse des ondes de volume se propageant dans le milieu considéré [12]. Les solutions de ces équations s'explicitent sous la forme de série de fonctions cylindriques. Pour chaque rang $n$ des séries solutions de l'équation (1), il faut déterminer 6 constantes. Celles-ci sont les inconnues d'un système linéaire et homogène issu des conditions aux limites à satisfaire en $r=a$ et $r=b$, conditions qui traduisent la continuité du déplacement radial, de la contrainte radiale et de la contrainte tangentielle. Soit $D_{n}$ le déterminant $6 \times 6$ du système des conditions aux limites. Ce système possède une solution non nulle si

$$
D_{n}(\omega, a, b)=0
$$

où $\omega$ désigne la pulsation de l'onde harmonique incidente. Les coefficients de ce déterminant [7] sont des fonctions affines des fonctions de Bessel $J_{n}$ et 
$Y_{n}$ ainsi que de leur dérivée par rapport à leur argument $x_{1}=k_{1} a$. Les entiers $n$ sont les modes de vibration ou résonances de la coque plongée dans l'eau ; à chaque fréquence de résonance est associée une onde circonférentielle se propageant à la périphérie du diffuseur [1].

Les fréquences des résonances sont obtenues à partir de (2) après application de la transformation de Sommerfeld-Watson. Le déterminant $D_{n}$ devient ainsi la fonction $D\left(\nu, x_{1}\right)$ par prolongement analytique dans le plan complexe de l'indice $n$ devenu la variable complexe $\nu$. L'équation caractéristique s'écrit :

$$
D\left(\nu, x_{1}\right)=0
$$

dont les racines sont habituellement notées $\nu_{l}\left(x_{1}\right)$. La condition d'existence d'une résonance est :

$$
\operatorname{Re}\left(\nu_{l}\left(x_{1}\right)\right)=n
$$

Pour $x_{1}$ réel positif $D\left(\nu, x_{1}\right)$ est une fonction continue de $x_{1}$. Les développements asymptotiques des fonctions $J_{\nu}, J_{\nu}^{\prime}, Y_{\nu}, Y_{\nu}^{\prime}$ utilisés pour évaluer les racines de (3) changent d'une zone à l'autre du plan complexe. De plus, pour le problème de diffusion, deux configurations physiques doivent être distinguées et le choix des développements des fonctions de Bessel diffère selon que la longueur d'onde incidente est très petite devant l'épaisseur de la coque ou de l'ordre de grandeur de cette dernière.

Le premier cas correspond à l'hypothèse des hautes fréquences $\left(k_{1} a, k_{1} b\right.$ et $\left.k_{1}(a-b) \gg 1\right)$ qui a été la plus étudiée jusqu'à ce jour. Les développements asymptotiques mis en œuvre sont du type développement de Debye et l'on obtient une infinité de racines $\nu_{l}\left(x_{1}\right)$. Lorsque l'on diminue la fréquence de l'onde incidente $\left(x_{1}\right)$, la longueur d'onde augmente et dès que celle-ci devient de l'ordre de grandeur de l'épaisseur de la coque il convient d'utiliser les développements de Lorenz (ou les développements en polynômes de Schöebe) [13, 14]. Les coefficients de $D\left(\nu_{l}, x_{1}\right)$ sont alors des polynômes dont le degré dépend de l'approximation retenue et les racines $\nu\left(x_{1}\right)$ de $(3)$ correspondant à cette deuxième situation sont en nombre fini. Cette seconde configuration physique se traduit sur le plan fréquentiel par l'existence d'une fréquence de coupure haute donnée par:

$$
x_{0}^{*}=\bar{K}(1-\eta)^{-1}
$$

où $\bar{K}$ représente un nombre d'onde moyen rapporté à celui des ondes se propageant dans le milieu 1 .

Pour l'ensemble des données expérimentales le tableau $\mathrm{V}$ présente la dernière fréquence observée pour l'onde $« l=0$ » et la borne théorique donnée par $x_{0}^{*}=\bar{K}(1-\eta)^{-1}$. Pour $\bar{K}$ il a été pris un rapport de nombre d'onde moyen estimé à partir des
Tableau V

\begin{tabular}{clr}
\hline$\eta$ & \multicolumn{1}{c}{$x_{0}^{*}$} & \multicolumn{1}{c}{$x_{\text {exp }}^{*}$} \\
0,67 & \multicolumn{1}{c}{3} & 1,81 \\
0,75 & 4 & 1,98 \\
0,85 & 6,67 & 7,20 \\
0,88 & 8,34 & 8,03 \\
0,93 & 18,08 & 17,30 \\
0,95 & 25 & 25,17 \\
0,97 & 37,9 & 44,38 \\
0,98 & 56,8 & 65,30
\end{tabular}

résultats expérimentaux. On note que l'accord est d'autant meilleur que la dernière fréquence réduite observée notée $x_{\exp }^{*}$ est faible. Ceci correspond au fait que le rapport $\eta$ n'est pas trop proche de 1 . Cependant, pour le tube tel que $\eta=0,95$ le résultat est bon et pour les tubes plus minces, un bon ordre de grandeur est obtenu.

\section{Calcul des fréquences des résonances.}

Les résonances de l'onde $« l=0$ » pour le tube tel que $\eta=0,85$ ont été obtenues après transformation de Sommerfeld-Watson par résolution de l'équation (3) dans le plan complexe $\nu$ (extension de l'indice entier $n$ ). Il est bien connu que la condition d'existence d'une résonance est donnée par (4).

La fréquence de la résonance est notée $x_{n l}$.

La largeur de la résonance est donnée par:

$$
\Gamma_{n l}=2 \frac{\operatorname{Im}\left(\nu_{l}\left(x_{1}\right)\right)}{\left[\mathrm{d} \operatorname{Re}\left(\nu_{l}\left(x_{1}\right)\right) / \mathrm{d} x_{1}\right]_{x_{1}=x_{n l}}} .
$$

Le couplage du tube avec le liquide externe est d'autant plus fort que la largeur de la résonance est grande. Aux basses fréquences, la recherche des résonances $\ll l=0$ » est effectuée par la résolution de (3) et (4). Lorsque la fréquence augmente, cette recherche devient difficile. Ceci est lié au comportement des fonctions de Bessel et aux propriétés de l'onde étudiée, en particulier le fait que le mode de vibration $n$ est toujours supérieur à l'argument de la fonction. Lorsque l'indice $n$ d'une fonction de Bessel est grand devant l'argument, la fonction de Bessel $J_{n}$ tend de façon exponentielle vers zéro d'où la difficulté de résoudre avec une bonne précision l'équation (4).

Cependant, lorsque la fréquence augmente, la recherche des résonances par la méthode de la matrice $S$ [1] est possible. L'utilisation de ce formalisme nécessite le choix d'un fond potentiel non résonnant. Lorsque la fréquence est suffisamment élevée, un fond potentiel rigide peut être choisi [15]. La détermination des fréquences de résonances peut alors être effectuée. L'approche des fréquences des résonances par la transformation de Sommerfeld- 
Watson ou par la méthode de la matrice $S$ est équivalente [1]. Afin de s'en assurer, pour un tube tel que $\eta=0,95$ et pour le mode $n=15$ des calculs numériques montrent que la fréquence de résonance, calculée par la transformation de Sommerfeld-Watson vaut 9,036, alors que par la théorie de la matrice $S$ elle vaut 9,037 . La concordance est donc très bonne. Pour l'ensemble des autres résonances, et parce que le calcul numérique est plus simple, la recherche des fréquences de résonances a été faite par la théorie de la matrice $S$.

\section{Discussion des résultats.}

Le calcul des fréquences des résonances $x_{n l}$ et des largeurs de ces résonances a été effectué pour les tubes étudiés expérimentalement. Les valeurs des paramètres sont les suivants :

$c_{\mathrm{L}}=6380 \mathrm{~m} / \mathrm{s}$ où $c_{\mathrm{L}}$ est la vitesse de phase des ondes longitudinales,

$c_{\mathrm{T}}=3100 \mathrm{~m} / \mathrm{s}$ où $c_{\mathrm{T}}$ est la vitesse de phase des ondes transversales,

$\rho=2790 \mathrm{Kg} / \mathrm{m}^{3}$ où $\rho$ est la masse volumique,

$c_{1}=1470 \mathrm{~m} / \mathrm{s}$ est la vitesse de phase du son dans l'eau.
Les résultats sont reportés dans les tableaux I-IV. L'accord entre les résultats théoriques et les résultats expérimentaux est très bon pour les tubes tels que $b / a=0,93 ; 0,95 ; 0,97$. En ce qui concerne le tube $b / a=0,98$ la différence est sans doute due à la difficulté d'enregistrer, dans le diagramme angulaire, tous les maxima : en effet, ceux-ci sont très rapprochés et comme il y a un petit angle mort dans la rotation du transducteur, il est possible qu'il y ait dans ce cas une erreur de une unité sur la valeur du mode $n$. Pour un tube de rapport $b / a$ donné, les largeurs des résonances augmentent avec la fréquence. Lorsque la fréquence tend vers zéro, la partie imaginaire est nulle; il n'y a plus couplage entre le tube et l'eau: on ne peut pas observer expérimentalement une telle résonance. Lorsque la fréquence croît, l'atténuation de l'onde circonférentielle croît également : au-delà d'une certaine fréquence la résonance ne pourra plus être observée expérimentalement. Ceci justifie l'existence des résonances $\ll l=0 »$ dans une certaine gamme de fréquence. Ce résultat avait déjà été mentionné par Breitenbach [16] à partir de résultats sur les fréquences de résonances uniquement. Cet effet de fenêtre est ainsi justifié.

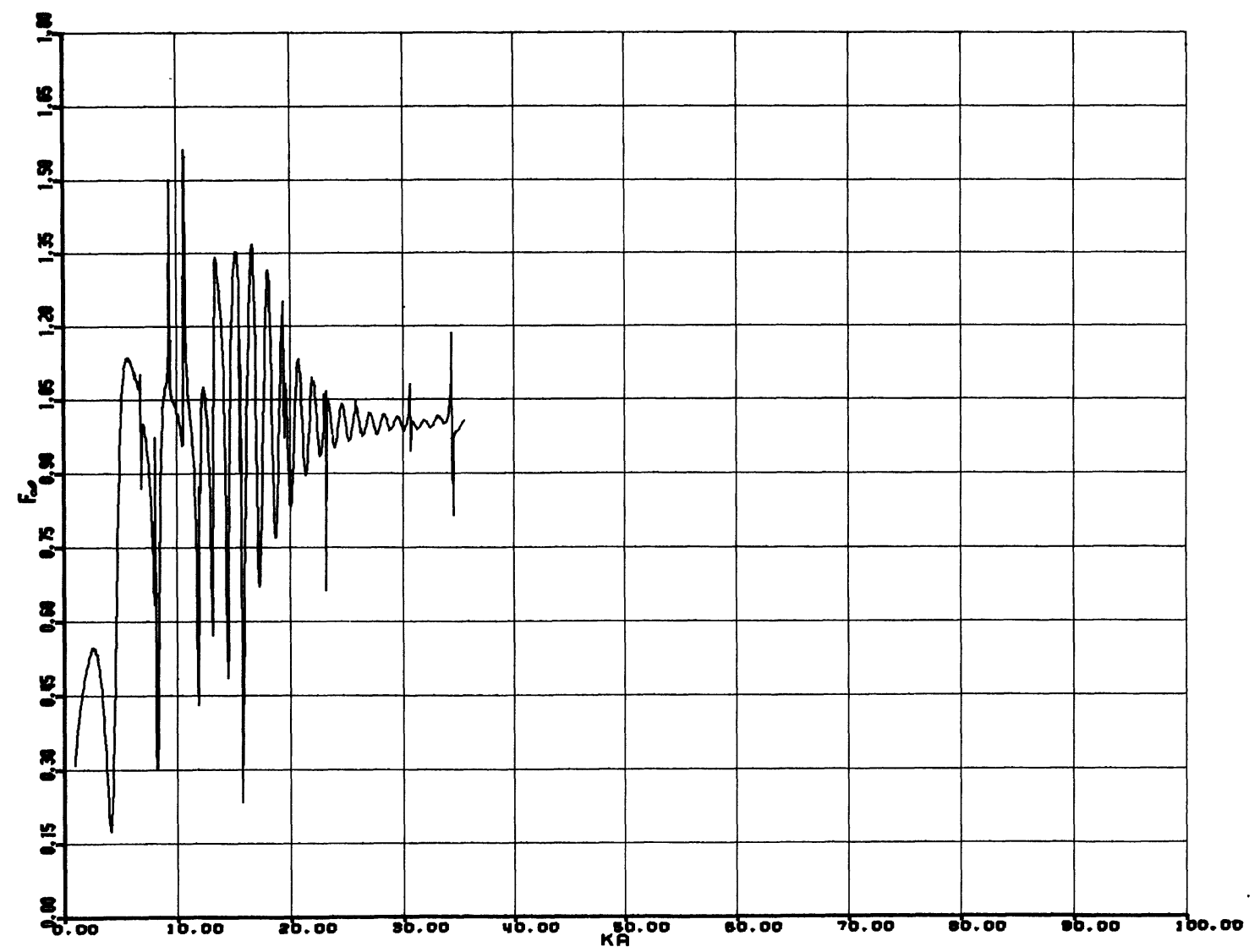

Fig. 11. - Tube $b / a=0,93$. Fonction de forme en champ lointain.

[Cylindrical shell $b / a=0.93$. Far field form function.] 


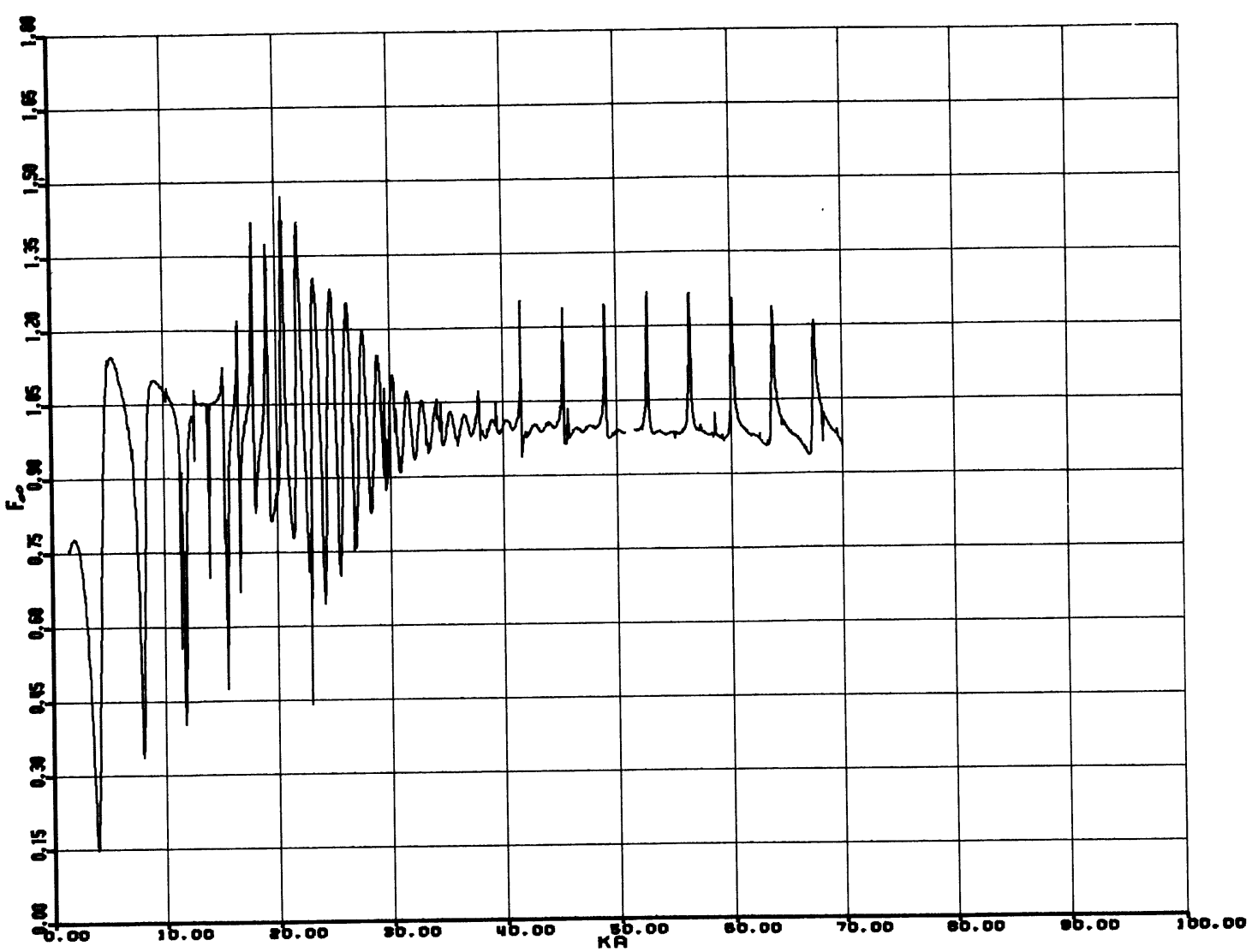

Fig. 12. - Tube $b / a=0,95$. Fonction de forme en champ lointain.

[Cylindrical shell $b / a=0.95$. Far field form function.]

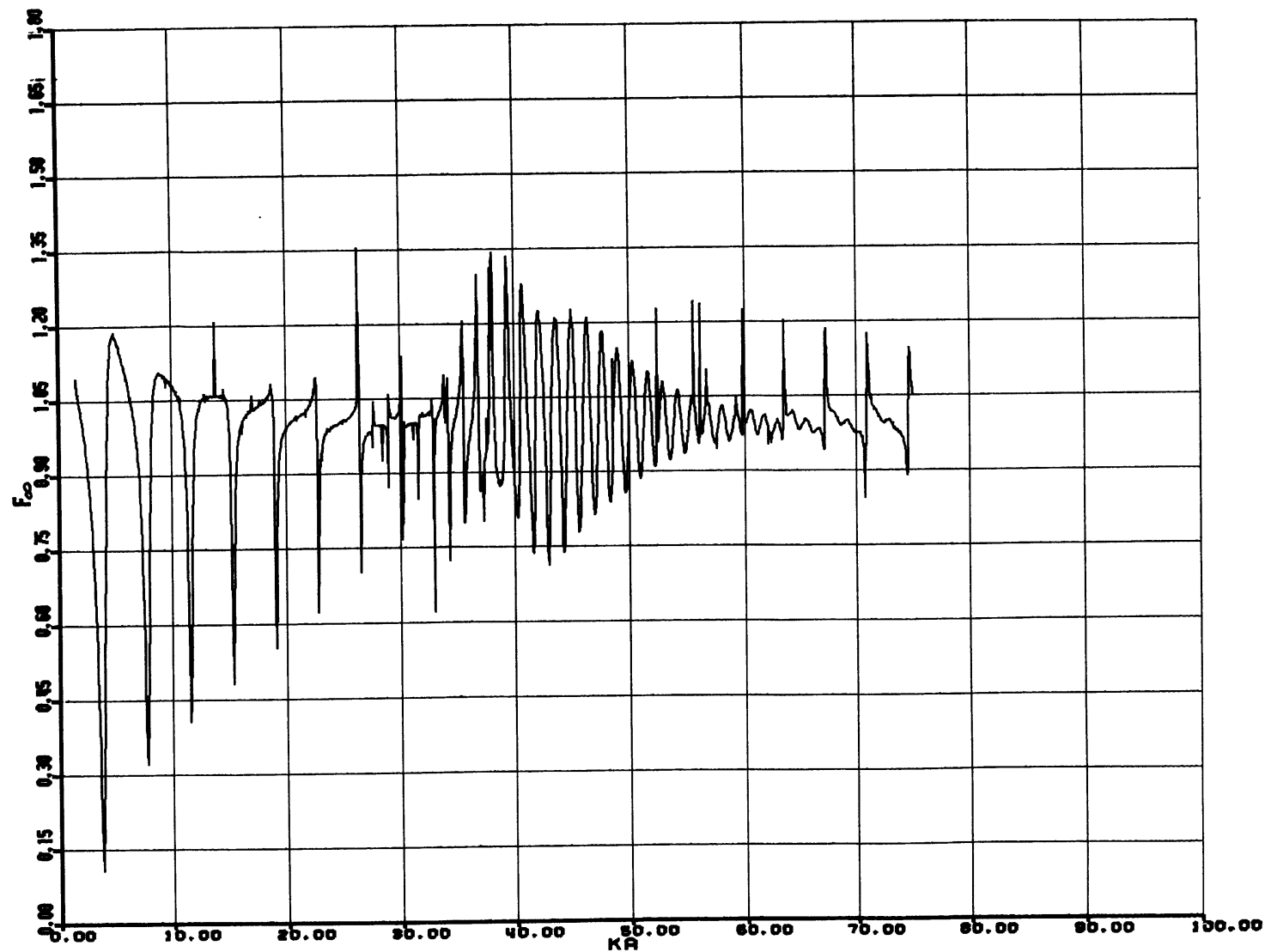

Fig. 13. - Tube $b / a=0,97$. Fonction de forme en champ lointain.

[Cylindrical shell $b / a=0.97$. Far field form function.] 


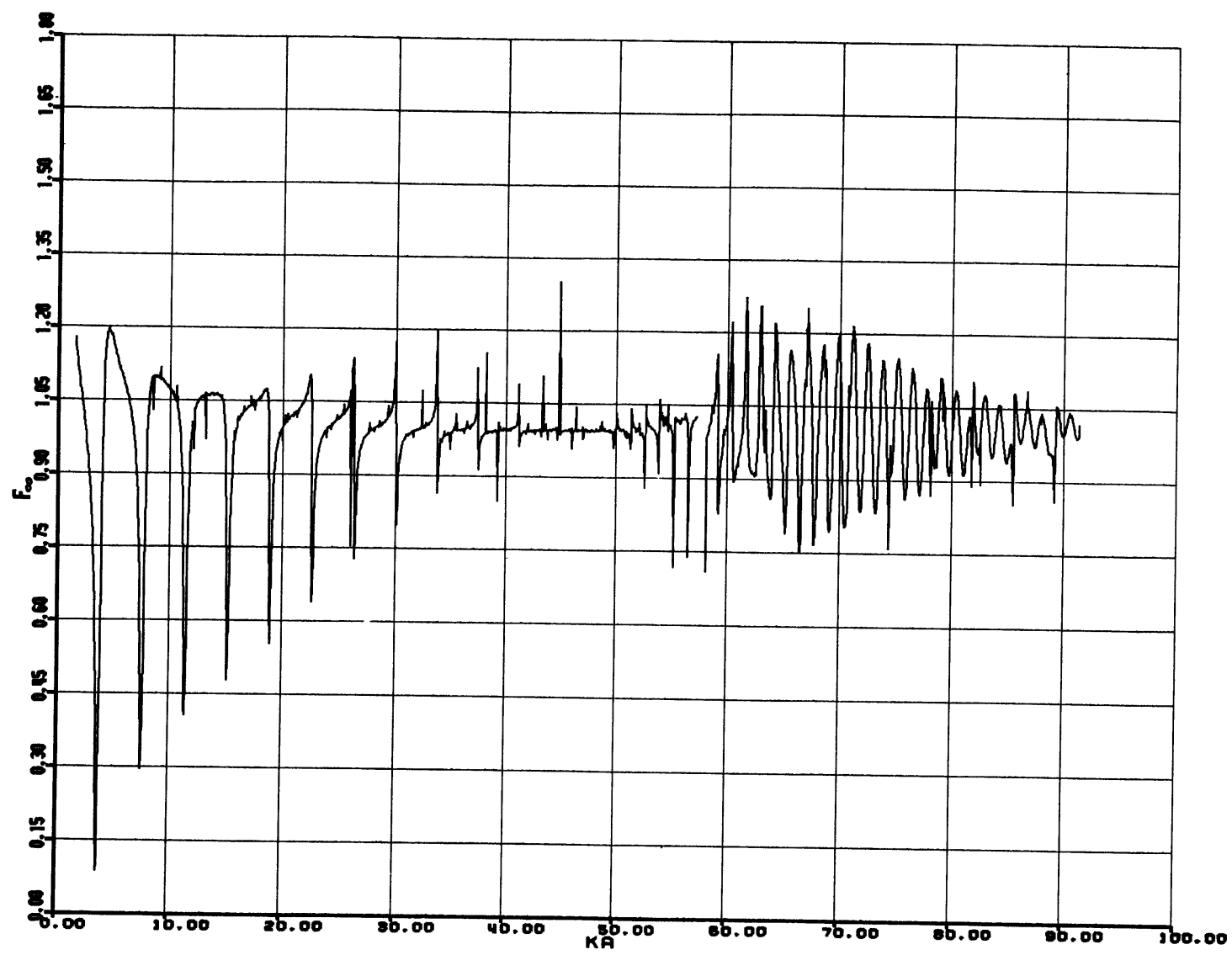

Fig. 14. - Tube $b / a=0,98$. Fonction de forme en champ lointain.

[Cylindrical shell $b / a=0.98$. Far field form function.]

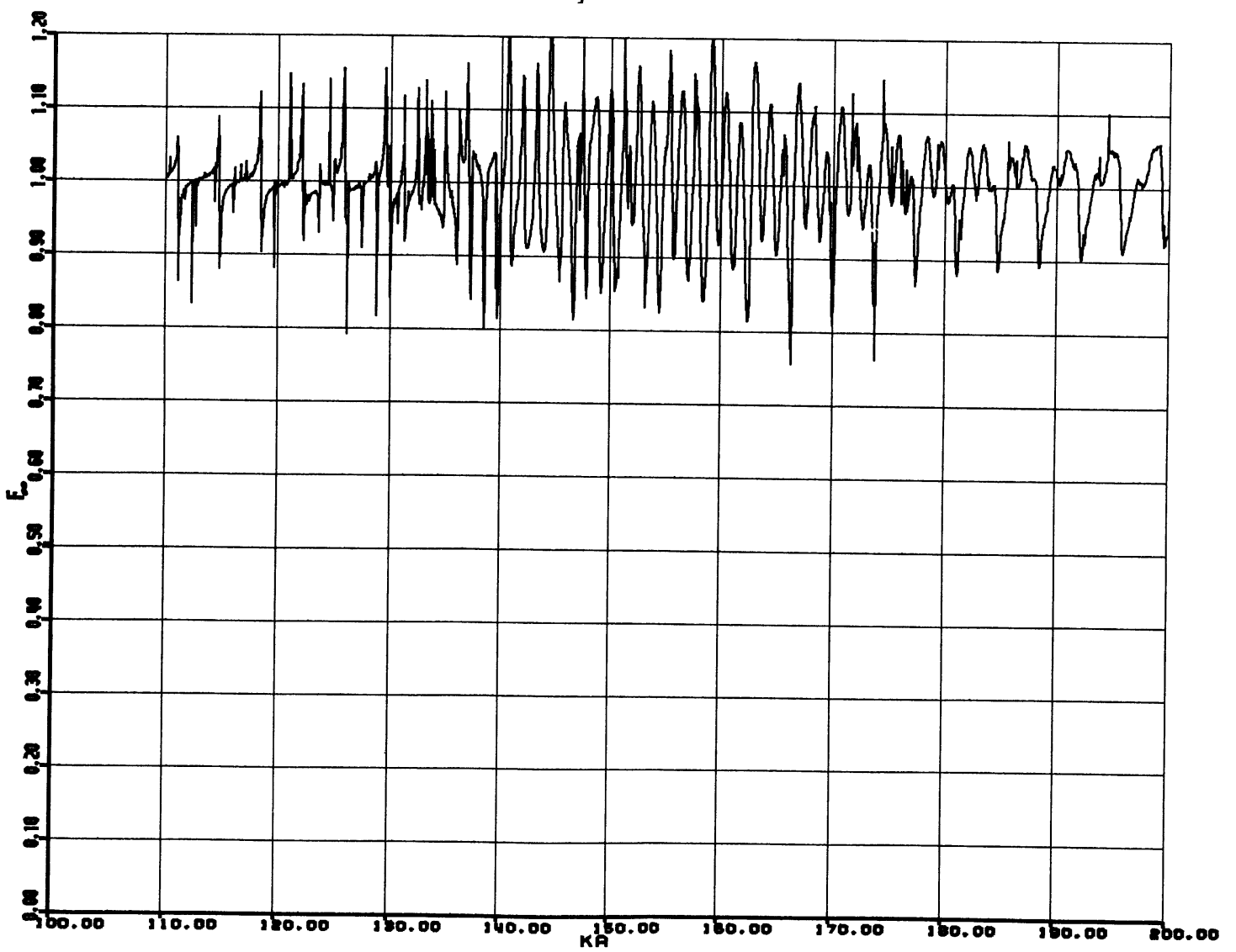

Fig. 15. - Tube $b / a=0,99$. Fonction de forme en champ lointain.

[Cylindrical shell $b / a=0.99$. Far field form function.] 
Afin de montrer l'effet de ces résonances sur la diffusion acoustique, la fonction de forme en champ lointain $F_{\infty}\left(x_{1}, \pi\right)$ [1] a été tracée pour chacun des tubes (Fig. 11-15). Un examen précis des courbes montre que les résonances se situent aux maxima de la fonction de forme. Pour les ondes de galerie à écho, les résonances correspondent en général aux minima de la fonction de forme. On remarque une bonne concordance, pour un tube donné, entre l'allure de $F_{\infty}$ et celle de la courbe expérimentale de la pression rétrodiffusée dans le régime forcé. L'effet des ondes $« l=0 »$ sur la fonction de forme en champ lointain est assez différent des ondes du type galerie à écho. On observe sur la courbe une succession de maxima et de minima assez régulièrement répartis, alors que pour les ondes de galerie à écho $l=2$ la courbe présente des variations brusques. Les courbes obtenues théoriquement sont analogues à celles publiées récemment par Veksler [17] ; cependant celui-ci a donné des valeurs erronées aux modes de vibration correspondant aux ondes $« l=0 »$. En effet, le mode est calculé à partir de la différence de fréquence entre deux résonances successives sans tenir compte du fait que le mode ne dépend pas linéairement de la fréquence. La vitesse de phase trouvée pour l'onde associée aux résonances est supérieure à la vitesse du son dans l'eau. Aussi l'auteur lie l'onde associée aux résonances précédentes à l'onde de Lamb $A_{0}$. Le calcul est en opposition avec nos résultats d'identification des résonances : la vitesse de phase est toujours trouvée inférieure à $c_{1}$.

Du point de vue théorique, afin d'interpréter les résultats concernant l'évolution de la vitesse de phase en fonction de la fréquence, l'onde «l $=0 »$ a été rapprochée des ondes de Lamb sur la plaque mince du type $A_{0}[5]$; elle a été également rapprochée des ondes du type Stoneley-Scholte [6]. Ces dernières ondes, dont la vitesse de phase est toujours inférieure à celle du son dans l'eau quelle que soit la fréquence, sont liées à la présence d'eau entourant la plaque. Aussi peut-on penser que les ondes $« l=0$ » seraient l'analogue, sur un tube, des ondes de Stoneley-Scholte. Des mesures de vitesse de groupe semblent confirmer ce point de vue [18].

Les auteurs remercient Gérard Géraut pour sa participation efficace aux calculs et à l'obtention des courbes théoriques.

Ce travail est soutenu par la Direction des Recherches Etudes et Techniques (DGA, Paris), contrats $86 / 045$ et $86 / 501$.

\section{Bibliographie}

[1] Flax, L., Gaunaurd, G. C., Überall, H., Physical Acoustics, XV (Academic Press, New York) 1980, 191-294

[2] Maze, G., Ripoche, J., Revue Phys. Appl. 18 (1983) 319.

[3] Derem, A., Rousselot, J. L., Maze, G., Ripoche, J., Faure, A., Acustica 50 (1984) 69.

[4] Maze, G., Ripoche, J., Derem, A., Rousselot, J. L., Acustica 55 (1984) 69.

[5] Rousselot, J. L., Acustica 58 (1985) 291.

[6] Izbicki, J. L., MAZE, G., Ripoche, J., Acustica 61 (1986) 137.

[7] IzBICKI, J. L., Thèse de Doctorat d'Etat, Le Havre (1986).

[8] Dragonette, L. R., Numrich, S. L., Franck, L. J., J. Acoust. Soc. Am. 69 (1981) 1186.

[9] Deprez, G., Hazebrouck, R., Rev. CETHEDEC 72 (1982) 73.
[10] De Billy, M., J. Acoust. Soc. Am. 79 (1986) 219.

[11] Delestre, P., Izbicki, J. L., MAZE, G., Ripoche, J., Acustica 61 (1986) 83.

[12] Voir e.g. VikTOROV, I. A., Rayleigh and Lamb Waves (Plenum Press, New York) 1967.

[13] Abramowitz, M., Stegun, I. A., Handbook of mathematical functions (Dover) 1965.

[14] Watson, G., A treatise on the theory of Bessel functions (Cambridge) 1966.

[15] Murphy, J. D., Breitenbach, E. D., Überall, H., J. Acoust. Soc. Am. 64 (1978) 677.

[16] Breitenbach, E. D., Überall, H., Yoo, K. B., $J$. Acoust. Soc. Am. 74 (1983) 1267.

[17] VeKsler, N. D., Wave Motion 8 (1986) 525.

[18] TALMANT, M. à paraître. 